of her HIV status until she was hospitalized for hepatic complications in June 1996. At that time, she had a high HIV RNA load and low CD4 counts.

No exposure to blood was evidenced. To test the hypothesis of a possible nurse-to-patient transmission, phylogenetic analyses were conducted using two HIV-1 genomic regions (pol reverse transcriptase [RT] and env $\mathrm{C} 2 \mathrm{C} 4$ ), each compared with reference strains and large local control sets (57 RT and $41 \mathrm{C} 2 \mathrm{C} 4$ local controls). Extensive analyses using multiple methodologies allowed the testing of robustness of phylogeny inference and assessment of transmission hypotheses. Results excluded nurse 1 and strongly suggested nurse 2 as the source of infection of the patient.

Because nurse 2 had advanced HIV disease, whereas the patient had signs of newly acquired infection, the researchers concluded that viral transmission occurred from the healthcare worker to the patient. Although the nurse was also infected with hepatitis $C$ virus, the patient showed no signs of hepatitis $\mathrm{C}$ virus infection. The mode of transmission was not identified.

FROM: Goujon CP, Schneider VM, Grofti J, Montigny J, Jeantils V, Astagneau P, et al. Phylogenetic analyses indicate an atypical nurse-to-patient transmission of human immunodeficiency virus type 1.J Virol 2000;74:2525-2532.

\section{Nosocomial Transmission of Mycobacterium bovis Bacille Calmette-Guérin}

A previous report of nosocomial infection due to Mycobacterium bovis bacille Calmette-Guérin (BCG) implicated contamination of chemotherapy solutions reconstituted under the same biosafety hood as BCG vaccine used for bladder-cancer therapy. Waecker and coinvestigators from the Naval Medical Center, San Diego, California, reported three similar BCG infections in children and described evidence of respiratory transmission to healthcare workers ( $\mathrm{HCWs)} \mathrm{from} \mathrm{one} \mathrm{patient.} \mathrm{These} \mathrm{children}$ were receiving chemotherapy for leukemia when they presented with active tuberculosis. Each isolate was identified biochemically and by both gas-liquid chromatography and major polymorphic tandem repeat-polymerase chain reaction. Pulsed-field gel electrophoresis showed that two isolates were identical strains and identical to the Tice and Connaught strains licensed in the United States for bladder chemotherapy. The third isolate differed by a single fragment after DraI restriction. One patient with heavily positive sputum exposed numerous HCWs. Of $41 \mathrm{HCWs}, 2(5 \%)$ converted their purified protein derivative skin test. These data underscored the risk of nosocomial BCG transmission by contamination of chemotherapy solutions and demonstrated the potential for transmission to $\mathrm{HCWs}$ from patients with active pulmonary disease.

FROM: Waecker NJ Jr, Stefanova R, Cave MD, Davis CE, Dankner WM. Nosocomial transmission of Mycobacterium bovis bacille Calmette-Guérin to children receiving cancer therapy and to their health care providers. Clin Infect Dis 2000;30:356-362.

\section{Contaminated Vials of Epogen}

Health professionals and dialysis clinicians have been notified by the FDA of problems with the multiple-use of Epogen vials (Amgen, Inc, Thousand Oaks, CA) labeled for single-use. Amgen was made aware of 21 episodes of bacteremia or pyrogenic reactions in patients receiving Epogen at a US dialysis unit. A CDC investigation revealed that unused portions of Epogen remaining in single-dose preservative-free vials were collected and pooled into common vials for use in other patients. These practices were linked to extrinsic bacterial contamination of Epogen.

The letter states that "... multiple entries should not be made into single dose vials, and residual medication from two or more vials should not be pooled into a single vial. As supplied, EPOGEN (Epoetin alfa) in single dose vials is a sterile solution. Although multidose vials with preservative are available, single dose vials do not contain a preservative. Once a syringe has entered a single dose vial, the sterility of the product can no longer be guaranteed."

\section{Low Risk of Transfusion-Associated Bloodborne Infections in England}

Regan and colleagues from the National Blood Service, London, England, recently reported the results of their ongoing study of recipients of 20,000 units of blood. Participants were adults who had been transfused recently. Patients had further blood samples taken at 9 months that were tested for markers of hepatitis B and C, HIV, and human $\mathrm{T}$ cell leukemia/lymphoma virus type I or II (HTLV) infections. Recent infections were distinguished from preexisting infections by comparison with blood samples taken before transfusion.

A total of 9,220 patients were recruited, and 5,579 recipients of 21,923 units of blood were followed up. No transfusion-transmitted infections were identified. Three patients acquired hepatitis B during or after hospital admission, but not through transfusion; $176(3 \%)$ had preexisting hepatitis B infection. Sixteen (0.29\%) patients had hepatitis $\mathrm{C}$, and $5(0.09 \%)$ had HTLV.

The authors concluded that the current risk of transfusion-transmitted infections in the United Kingdom is very small, although hospital-acquired infections may arise from sources other than transfusion. A considerable proportion of patients have preexisting infections.

FROM: Regan FA, Hewitt P, Barbara JA, Contreras M. Prospective investigation of transfusion transmitted infection in recipients of over 20,000 units of blood. TTI Study Group. BMJ 2000;320:403-406.

\section{Safety and Cleaning of Medical Materials and Devices}

Merritt and coinvestigators from the FDA, Center for Devices and Radiological Health, Division of Life Sciences, in Rockville, Maryland, conducted a study to evaluate dif- 